\title{
A simulation-based LCC model for railway track
}

\author{
Wenxu Li* and Richard Dwight** \\ *University of Wollongong, Australia,wenxuli@uow.edu.au \\ *University of Wollongong, Australia, radwight@uow.edu.au
}

\begin{abstract}
Because of the complexities of the railway track system compositions and their degradation mechanisms, the existing models built for relevant LCC estimation are either too simplified or focused on a part of the system. In this paper, a simulationbased model is proposed which is to incorporate all of the major track components such as rail, ballast and sleepers. A cost breakdown methodology is adopted to estimate the cost on a component-by-component and activity-by-activity basis. The interactions of track components and between maintenance and track components can be considered during the simulation process. Some activities whose implementation is purely experience-based can be loosely added as extra cost elements by disregarding its connection with other activities. Though attempting to embrace all, the simulationbased model is still considered easier to implement and potentially faster to run compared to the more complex and probably more powerful models including the PetriNet models: the tools for its development are readily available. Apart from that, only knowledge on Monte Carlo simulation is required. In addition to providing a tool for the planning of maintenance actions, the model may also be used to evaluate different track form options. By replacing the simulation-based models with parametric models or limiting the focus to one or several track components, the model can be simplified conveniently.
\end{abstract}

\section{Introduction}

Life Cycle Cost, LCC, by evaluating the whole of life cost of a system, provides a reliable measure to support various decisions. By ranking costs on either a componentor activity-basis problematic components in a system or weak spots in a maintenance regime can be identified, which may in turn facilitate design optimization and resource utilization maximization. In the railway sector, LCC has been used to optimize maintenance strategies (Meier-Hirmer et al., 2009, Caetano and Teixeira, 2015), compare track forms (Pratico and Giunta, 2018) and evaluate track performance (Rama and Andrews, 2016). Breakdown of LCC to a level where cost for each element is accessible and then aggregated is the usual approach to calculate LCC (ISO 15686-5, 2017).

The major cost for managing a railway track during the operation stage arises from maintenance driven by the degradation of track components. Maintenance and renewal in turn affect degradation development of these components. Knowledge of degradation states of track components and restoration effects of maintenance and renewal activities on these components therefore plays a central role in an attempt to understand how the cost is derived.

Degradation modelling of a railway track therefore needs to address: 1) multiple interacting components: each track is made of a superstructure (the part above ballast or concrete track slab) and a substructure (the rest of the track after excluding the 
superstructure), each consisting of various components; 2) randomness of the degradation state of a component and the restoration effect of a maintenance action on a component: different degradation growth trends are reported: linear (Vale et al., 2012, Andrews, 2013) and exponential (Quiroga and Schnieder, 2012). Andrade and Teixeira (2011) observed different track degradation rates at sites of seemingly same conditions based on the Austrian context. The restoration effect of tamping is reported to be tamping history dependent: the more tamps perfomed, the less effective it becomes, partially due to ballast damage caused by tamping operation (Quiroga and Schnieder, 2012); and, 3) variation of maintenance design and scheduling across organizations.

This work sets out to develop a degradation-based LCC model to allow LCC performance evaluation of a track form, comparison between different track forms and exploration of various maintenance strategies. Starting with a literature review to identify current progress on track degradation modelling, the work proposes a simulated-based LCC model in which degradation models for major track components are developed. The framework and theoretical basis of the model are then introduced.

\section{Track degradation modelling - a review}

\subsection{Track degradation measures}

Degradation within a railway track system results in the loss of system integrity caused by failures and wear of track components and losss of track geometry. The indicators used to represent component degradation level may vary with failure mechanisms and manifestations. A rail, for example, may develop corrugation which is measured by the size of a corrugation area or suffer from rolling contact fatigue which is characterized by crack size. Similarly, various measures to indicate track geometry have been developed. The two most widely used are the horizontal alignment, or 'level', which measures the waviness of the track in the travelling direction (Andrade and Teixeira, 2015) and the longitudinal level, or 'top', which measures the deviation of rail top surface from the ideal position (Vale et al., 2012, Quiroga and Schnieder, 2012, Andrews, 2013, Haigermoser et al., 2014). The two meaures are also illustrated in Figure 1. Track quality indices, TQIs, which are a combination of some of the individual indicators, have also been developed, but the result can be misleading, especially when poor values for individual measures are disguised under a good overall TQI. Deviation of any measure from the acceptable level requires correction. 


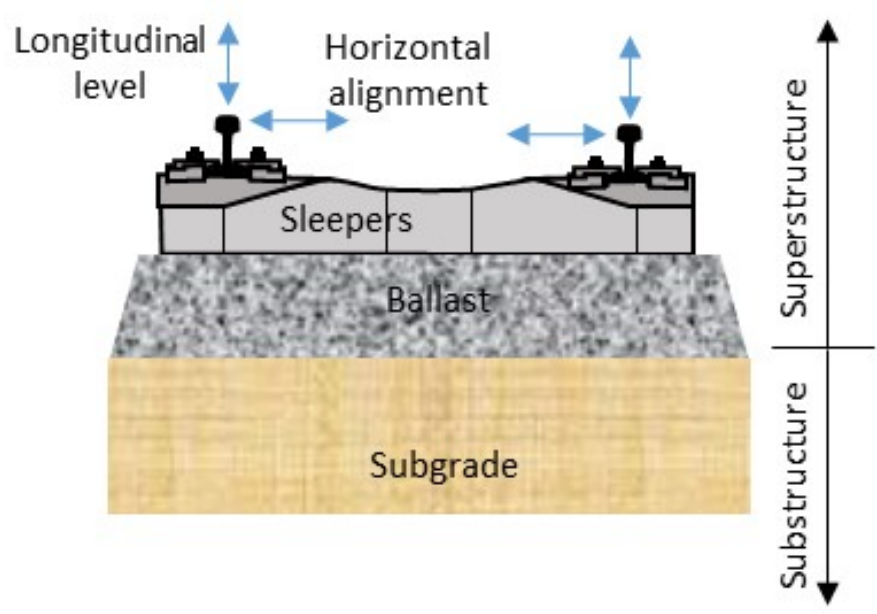

Figure 1. Horizontal alignment and longitudinal level for a ballasted track

\section{Degradation modelling}

Degradation models developed mostly focus either on a single track component or several but assumed independent track components. The components focused, the maintenance actions allowed and the failure mechanisms investigated are often limited. The model is either deterministic or statistical. Deterministic models are built from the perspective of the underlying degradation mechanism and model a track degradation indicator as a function of explanatory variables, such as speed, load, age, etc. In contrast, statistical models approach the problem from the data viewpoint only. The objective of such a study is to obtain the distribution for the time taken to reach a specific state of degradation. The models are arguably more in line with the random nature of observed track degradation states.

Examples of deterministic models for track degradation include Zhang et al. (2000) and Michael (1985). Michael (1985) built a system based on the engineering economics approach and the degradation states of track components. This model allows the consideration of the various service options and evaluation of alternatives in equipment, operating plans, track structure and maintenance standards. The degradation rates of track components are related to the flow of the traffic, i.e. tonnage, enabling the remaining service life of each component to be estimated. Only renewal is considered as the maintenance option in the model.

Zhang et al. (2000) developed an ITDM (Integrated Track Degradation Model) where rail, sleeper, ballast and subgrade are all taken into account. Through structural analysis on each component, the degradation state of each component is established as a function of time and traffic loading condition. For rail degradation, only wear is considered. Interaction of the various components is also allowed. For instance, to account for the impact of differing track qualities on sleeper crack development, the loading a sleeper is subject to is adjusted accordingly. Ahac and Lakušić (2015) built a mechanistic-empirical model for gauge degradation on the tram track. Guler et al. (2011) conducted a review on the development of mechanistic degradation models for track geometry. Dahlberg (2001) surveyed the mechanistic models on track settlement. Despite being easy to implement and understand, as pointed out in Andrews (2013), 
there is a possibility of invalidating this type of model completely if the dominant causal variable is missed out during modelling.

Earlier work on statistical modelling includes Esveld (1990) who identified the necessary information for assessing degradation states of various track components. Track geometry data together with maintenance limit values are used to demonstrate the estimation of best timing for applying tamping. The most severe measure is always used for track geometry evaluation. The track condition at a section/network level is condensed from those at a unit level where data is collected, e.g., $200 \mathrm{~m}$ as covered by a track maintenance vehicle each time. Mixed normalized track quality indicators are used to project track degradation level.

More recent developments include Quiroga and Schnieder (2012) who developed a statistical model to describe both the degradation process and the restoration process achieved by tamping interventions on one track section. Only longitudinal level is concerned. An exponential growth law is assumed. Both the initial degradation level and the power in the exponential law are represented by independent lognormal distributions and assumed to depend on the number of tamps since last renewal.

Overall, the review tends to show that track degradation models based on deterministic approaches have diminished over time. This has been overtaken by datadriven approaches, partly attributed to the growing affluence of condition data in the railway industry. Techniques to analyse big data have been brought in. Guler (2014) used neural networks to investigate track settlement under a Turkish context. Instead of combing all of the track geometry indicators into one, Lasisi and Attoh-Okine (2018) used principal components and afterwards machine learning to verify the performance of using principal components to evaluate the track geometry condition.

Stochastic processes, on the other hand, by being able to take into account various uncertainties, seem to coalesce with the degradation processes. Various applications have been attempted. It is given the particular attention in the following text.

\section{Statistical modelling on track degradation}

\section{Petri nets}

Andrews (2013) utilised the Petri Net, PN, where the deterioration, maintenance and inspection processes for a track section are all integrated. The model was developed for a track section at a length of $1 / 8$ miles $(0.2 \mathrm{~km})$, which is the unit length for track monitoring on the UK rail network. Only longitudinal level is investigated. Inspections, ballast tamping and renewal are the maintenance actions included. No cost is estimated though the relevant parameters for estimating the cost are readily available from the outputs of the model, such as the number of interventions and the durations of interventions resulting from speed restriction and line closure. The track degradation level is represented by four discrete states: new (a renewal will restore the track to a condition as good as new), condition needing maintenance; condition requiring speed restriction and condition needing line closure. The time distributions to reach a given state of degradation are obtained from historical measurements. To address the limitation of insufficient data required for the determination of the model parameters, 
the track is segmented into small sections and the degradation process after each renewal is treated separately. By varying the rules for carrying out renewals, such as a predetermined lifetime, a maximum number of interventions and an intervention interval too short, and inspections, e.g., at a specified intervention interval, various combinations of renewal and inspection policies can be explored through Monte Carlo simulation. It is noted that the model does not provide an optimal maintenance solution other than a performance evaluation of a given solution.

Since then the model has undergone substantial development. Prescott and Andrews (2013) presented a framework where track models at a regional scale are built by integrating section-based models. Both stone-blowing and tamping are included as possible maintenance actions, but ballast renewal is excluded. Resource limitations such as the number of tampers and stone-blowing machines, are allowed. Opportunistic maintenance is enabled. The geographical locations of track sections which are important for opportunistic maintenance are missing in the model, but maintenance on a section is more likely to take advantage of an opportunity arising from the adjacent sections than otherwise. Kilsby et al. (2019) applied PN to the overhead line equipment (OLE). Rama and Andrews (2016) introduced new features such as emergency restriction zone and line closure zone at the network level in their model which thus resembles more the reality. LCC was also introduced based on categorical decomposition. Both opportunistic and concurrent maintenance are enabled in the model. Their case study shows that if situations allow, combination of both maintenance strategies provides better performance.

However, none of the work on PN has attempted to address track degradation issues using track geometry indicators other than longitudinal level. The major bottleneck of PN-based models is that it is complex to implement which requires independent development especially when self-defined features are introduced. The computation is also very demanding. Determination of the distribution of time to reach a degradation state requires significant amount of data which may be difficult to acquire in practice. Yianni et al. (2018) proposed a method to accelerate the speed of PNs by taking advantage of the power of graphic units. Until these barriers are removed, a wide scale of applications of PNs outside laboratories seems still far away.

\section{Stochastic process-based models}

Markov process (Shafahi and Hakhamaneshi, 2009, Prescott and Andrews, 2015, Sharma et al., 2018) utilises a set of discrete states, corresponding to different levels of track degradation. Normally only stationary Markov process is considered in these models. This limits the applicability of the model given that trending transition rates may be required to adequately model the situation of track degradation. A separate model has to be developed each time a new section is introduced. A model of a prohibitively large size is unavoidable when a number of sections are considered. Shafahi and Hakhamaneshi (2009) developed a model based on the 'Combined Track Record Index' which is an aggregated parameter over all of the measured track geometry indicators in their Markov model. Sharma et al. (2018) instead applied a data mining technique: 'random-forest', to estimate the occurrence rate of geo-defects (isolated defect requiring immediate repair).

The Markov model of Prescott and Andrews (2015) was section-based. By limiting the maximum allowable number of tamps on a section to 7 , the model defines four 
states for each growth path between two successive tamps: good, critical (maintenance required), speed-restriction and line-closure. Each deterioration process between successive tamps is treated as an independent phase. By further introducing an intermediate state after tamping which represents an improved, but not as 'good as new' state, as the initial state of the following phase, the whole degradation process from one ballast renewal event to the next can be modelled. The inspection scheme is introduced by allowing each state to have two variants: known, i.e. a condition revealed by inspections; and unknown, i.e. an unrevealed condition predicted by the model. Different maintenance strategies, such as: different inspection intervals; renewal intervals; and, geometry thresholds to initiate tamping, are explored.

Meier-Hirmer et al. (2006) used gamma process to model the deterioration process of track longitudinal level. Gamma process is a non-decreasing process which has been used intensively to model structure degradation including wear (van Noortwijk et al., 1992, van Noortwijk, 2009). A linear growth law is assumed and included in the shape parameter of the underlying gamma distribution.

In front of the ever-increasing data, Bayesian theory seems able to make the best use of existent data to determine model parameters whereas allow the use of new data to update these parameters whenever they come in. In combination with stochastic processes such as the Winer process and the gamma process, it can be used to model the degradation process of many structures.

The hierarchical Bayesian approach, by extending the fundamental Bayesian theory to allow model parameters, $\boldsymbol{q}$, conditioned on data, $\boldsymbol{y}$, to be further dependent on $\boldsymbol{f}$, often called hyperparameters, provides a mechanism for structure correlation. In other words, the joint conditional posterior distribution of $\boldsymbol{f}$ and $\boldsymbol{q}$ is given by

$$
p(\boldsymbol{f}, \boldsymbol{q} \mid \boldsymbol{y})=\frac{p(\boldsymbol{f} \mid \boldsymbol{q}) p(\boldsymbol{q}) p(\boldsymbol{y} \mid \boldsymbol{q})}{\dddot{\boldsymbol{\omega}} p(\boldsymbol{f} \mid \boldsymbol{q}) p(\boldsymbol{q}) p(\boldsymbol{y} \mid \boldsymbol{q}) d \boldsymbol{q} d \boldsymbol{f}} \mu p(\boldsymbol{f} \mid \boldsymbol{q}) p(\boldsymbol{q}) p(\boldsymbol{y} \mid \boldsymbol{q})
$$

from which the marginal conditional distribution of $\boldsymbol{q}$ can be integrated. Similarly $\boldsymbol{f}$ can be dependent on other variables.

Maes (2013) applied the hierarchical Bayesian approach together with Gaussian process to model the depth growth process of pipeline corrosion pits. By assuming independent means but shared scale parameters among these Gaussian distributions, both uniqueness of and interactions between different pits are enabled. Other application examples of hierarchical Bayesian approach include (Zhang et al., 2014, Al-Amin et al., 2014, Zhang, 2014). The approach has also been similarly applied in stochastic processes such as geometrical Brownian motion (Zhang and Zhou, 2015) and inverse Gaussian process (Zhang et al., 2013), both for pipeline degradation modelling.

Despite the availability and wide applications of hierarchical Bayesian models and the supporting simulation tools, its application in the railway sector for modelling track geometry is very limited. Andrade and Teixeira (2013) developed a section-based degradation model for longitudinal level based on the Gaussian process. Ballast tamping is the only maintenance action considered. The mean of the underlying 
Gaussian distribution, $m$, is assumed to have a linear relationship with the initial condition, $a$, and the track usage, i.e. tonnage, $\mathrm{T}$, by

$$
m=a(1+d)^{N}+b \mathrm{~T}
$$

where $b$ is the degradation rate. To account for the restoration effect of tamping, a factor $d$ is introduced. By limiting $d>0$, the track geometry condition after a tamping is guaranteed to be worse than the one from the previous tamping. $N$ denotes the number of tamps in history. To further account for the renewal effect, two separate sets of initial condition and growth rate, i.e. $a$ and $b$ are introduced for non-renewed and renewed tracks, respectively.

To enable spatial correlation between neighbouring sections, the initial condition of a section is assumed to consist of two components: one is common to all the sections on a segment; the other is dynamic allowing for variations across the sections. The dynamic term behaves like a random walk from the mean variation which is an average over all the neighbouring sections of a section of interest. Andrade and Teixeira (2015) later extended the model to include the horizontal alignment as well. The two geometry quality indicators are assumed to be independent of each other.

In Andrade and Teixeira's models, the way of including the effect of tamping is debatable as no prior evidence supports the relationship, $(1+d)^{N}$. It is also hard to introduce additional maintenance actions other than tamping within the current framework. Despite all of these drawbacks, by being simple and allowing for integration of certain levels of uncertainties, the model seems advantageous than many which are currently available.

It is noted that in order to implement Bayesian models, informative priors for the model parameters are valuable by reducing the sampling space of these parameters and thus improving the searching or converging speed. This requires some knowledge about these parameters before a simulation starts. Otherwise, non-informative priors, e.g., an inverse gamma distribution with a small variance and a flat prior, are required. One benefit of using gamma distribution as the prior is that it belongs to the conjugate family, i.e. the posterior distribution also follows gamma distribution thus providing an explicit formulation. Elicitation of expert judgement to determine the priors by the use of Bayesian analysis (van Noortwijk et al., 1992) has also been available.

A list of the track degradation models is given in Table 1, summarising the pros and cons of these reviewed models. In summary, most of the models are targeted at ballasted track despite being generic mathematically. With the availability of track condition and maintenance data, data-driven approaches are becoming more prevalent. Among them, PN-based approaches seem to be the most comprehensive by being able to unite degradation, intervention and inspection processes as well as life cycle cost estimation all under one framework. Hierarchical Bayesian models, while allowing the spatial interaction of track components to be taken into account, are also able to maintain the simplicity of implementation. 
Table 1. Review of the track degradation models.

\begin{tabular}{|c|c|c|c|}
\hline Methods & Pros & Cons & $\begin{array}{l}\text { Example } \\
\text { Literature }\end{array}$ \\
\hline Petri Nets & $\begin{array}{l}\text { It is modular, which allows } \\
\text { models at various levels of } \\
\text { granularity to be built; it has } \\
\text { great flexibility, which } \\
\text { allows the users to develop } \\
\text { customized functions (places } \\
\text { and transitions in the terms } \\
\text { of PN); it allows comparison } \\
\text { of various maintenance } \\
\text { options including } \\
\text { opportunistic and concurrent } \\
\text { maintenance and parameter } \\
\text { sensitivity analysis. }\end{array}$ & $\begin{array}{l}\text { Implementation of the models is } \\
\text { difficult: add-on features have to be } \\
\text { developed independently given no } \\
\text { commercial software is available; } \\
\text { the scale of the model increases } \\
\text { significantly with the length of the } \\
\text { track modelled and the } \\
\text { computation time may be } \\
\text { excessive; optional decisions are } \\
\text { all prescribed through defining the } \\
\text { firing rules of the transitions in PN. } \\
\text { As a result, it cannot generate a } \\
\text { decision option for the users. }\end{array}$ & $\begin{array}{lr}\text { Andrews } & (2013), \\
\text { Rama } & \text { and } \\
\text { Andrews } & (2016), \\
\text { Prescott } & \text { and } \\
\text { Andrews } & (2013), \\
\text { Kilsby } & \text { et al. } \\
(2019) & \end{array}$ \\
\hline $\begin{array}{l}\text { Bayesian } \\
\text { Approach }\end{array}$ & $\begin{array}{l}\text { A model can be updated with } \\
\text { new information; spatial } \\
\text { correlation can be } \\
\text { considered; easier to } \\
\text { implement than PN; models } \\
\text { can be built at a network } \\
\text { level. }\end{array}$ & $\begin{array}{l}\text { It only allows evaluation of } \\
\text { decision thresholds; simple } \\
\text { degradation laws have to be used; it } \\
\text { may still be difficult to implement. }\end{array}$ & $\begin{array}{lr}\text { Andrade } & \text { and } \\
\text { Teixeira } & (2013), \\
\text { Andrade } \quad \text { and } \\
\text { Teixeira (2015) }\end{array}$ \\
\hline $\begin{array}{l}\text { Markov } \\
\text { model }\end{array}$ & $\begin{array}{l}\text { Easy to implement; results } \\
\text { easy to interpret. }\end{array}$ & $\begin{array}{l}\text { Track degradation level is } \\
\text { discretised so it is unable to provide } \\
\text { a full picture of exact track } \\
\text { degradation level at a time; the size } \\
\text { of the model increases significantly } \\
\text { with the number of states; only the } \\
\text { homogeneous model has been } \\
\text { trialled by far; state sojourn time is } \\
\text { exponential. }\end{array}$ & $\begin{array}{l}\text { Sharma et al. } \\
(2018), \quad \text { Shafahi } \\
\text { and } \\
\text { Hakhamaneshi } \\
(2009), \quad \text { Prescott } \\
\text { and Andrews } \\
(2015)\end{array}$ \\
\hline $\begin{array}{l}\text { Artificial } \\
\text { Neural } \\
\text { networks }\end{array}$ & $\begin{array}{l}\text { Relations between various } \\
\text { variables can be studied. }\end{array}$ & $\begin{array}{l}\text { Calculation processes are hidden; } \\
\text { results may be hard to interpret. }\end{array}$ & Guler (2014) \\
\hline $\begin{array}{l}\text { Gamma } \\
\text { process }\end{array}$ & $\begin{array}{l}\text { It allows for uncertainties of } \\
\text { track degradation process; it } \\
\text { is non-decreasing, agreeing } \\
\text { with a track degradation } \\
\text { process. }\end{array}$ & $\begin{array}{l}\text { Only linear models have been } \\
\text { trialled to model the track } \\
\text { longitudinal level deterioration; No } \\
\text { account is taken of previous } \\
\text { maintenance: e.g., tamping, or } \\
\text { influence of other factors. }\end{array}$ & $\begin{array}{l}\text { Meier-Hirmer et } \\
\text { al. (2006) }\end{array}$ \\
\hline
\end{tabular}




\section{Simulation-based Model}

Based on the review, the proposed degradation models making up the core of the LCC model aim to address:

1) interaction of track components,

2) impact of maintenance actions on track components,

3) ability to simulate different maintenance management assumptions: in the form of possible ill-advised budget constraints,

4) track forms with different configurations,

5) various maintenance strategies such as opportunistic and concurrent maintenance, preventive maintenance and corrective maintenance,

6) degradation models of different track components altogether; and

7) different sizes of track, e.g. a section vs. a network.

\section{LCC model overview}

The LCC model targets track systems and is segment-based. Models for tracks at a larger scale are obtained by assembling the segment-based models. Degradation models developed are component-based. Auxiliary systems such as the signalling systems and overhead wiring system are not included, but they can be incorporated as components.

In general, there are five cost elements which make up the LCC of a track, as listed in Table 2. These may be further distilled into elements of primary and secondary importance. The primary elements may include construction cost and maintenance cost. Disposal cost and externality cost are considered secondary and excluded in the model. They may become prominent with the reduction of the primary costs. By then their contributions can be included separately. It is noted that models to address secondary cost elements, esp. externality cost related to carbon emission, have been available (Pratico and Giunta, 2018). The amount of carbon emission over the life-cycle of a railway track has been studied by Milford and Allwood (2010).

Table 2. LCC Components

\begin{tabular}{|c|c|l|}
\hline $\begin{array}{c}\text { Cost } \\
\text { Element }\end{array}$ & Included & \multicolumn{1}{|c|}{ Description } \\
\hline $\begin{array}{c}\text { Construction } \\
\text { cost }\end{array}$ & $J$ & $\begin{array}{l}\text { The initial capital investment on building the } \\
\text { infrastructure. It is considered the major contributor of the } \\
\text { acquisition cost. }\end{array}$ \\
\hline $\begin{array}{c}\text { Maintenance } \\
\text { cost }\end{array}$ & $J$ & $\begin{array}{l}\text { The cost spread over the whole life of a railway track and } \\
\text { incurred by the necessity to maintain the serviceability of } \\
\text { the track and ensure the safety of traffic operations }\end{array}$ \\
\hline $\begin{array}{c}\text { User impact } \\
\text { cost }\end{array}$ & $\zeta$ & $\begin{array}{l}\text { Cost related to penalty regime imposed by the train } \\
\text { operators for the unavailability of the track including speed } \\
\text { restriction and track closure }\end{array}$ \\
\hline $\begin{array}{c}\text { Disposal } \\
\text { cost }\end{array}$ & \} & $\begin{array}{l}\text { The cost that has to be paid in order to dispose of the retired } \\
\text { material and can be positive, or negative(income) }\end{array}$ \\
\hline Externality & \} & $\begin{array}{l}\text { Costs related to adverse environmental impact, such as } \\
\text { noise and vibration; CO2 and other health-affecting gas } \\
\text { emissions and pollutant discharges }\end{array}$ \\
\hline
\end{tabular}


The cost related to the train operation is also excluded. However, the penalty cost imposed on the track owner/operator by train operators due to service provision disruption is included. The cost corresponds to the user impact cost in Table 2, determination of which requires knowledge of the underlying penalty regime.

Present Value, PV, is used to represent the LCC. It can be conveniently converted into other cost measures such as Future Equivalent Amount, Annual Equivalent Amount, and Internal Rate of Return (Fabrycky and Blanchard, 1991).

Both the inflation rate, $i$, and the discount rate, $r$, are taken into consideration, i.e.

$$
P V=\mathrm{a} \frac{c_{t}(1+i)^{t}}{(1+r)^{t}}
$$

where $c_{t}$ is the nominal cost at time $t$.

Construction cost model

Construction cost is traditionally, if perhaps inappropriately, the expenditure most evaluated when making an investment decision, given its visibility and immediacy. It is also a significant contributor to LCC for both slab and ballasted track (Garry Thuer and Vocks, 2012). If not directly available, the construction cost may be assembled from the following four components:

- material cost

- labour cost

- transportation cost

- equipment cost

Material cost may be inferred from the prices of the compositions (Swei et al., 2017). The average labour cost rate may be obtained from the labour market statistics. The transportation cost is particularly relevant when precast slabs are used in the construction as they are often constructed off-site in a workshop. Given the weight and size of a slab and the capacity of a truck as well as the distance between the workshop and the construction site, the number of journeys may be worked out leading to a relatively accurate estimate of the transportation cost. The same method may be applied to the transportation of raw materials for the construction of other track forms. Equipment cost is concerned with the lease fee of the construction equipment and may also be accessed from the market statistics.

\section{Maintenance cost model}

The maintenance cost model proposed is simulation-based by integrating degradation models of track components with a defined maintenance regime. At each time step of the simulation, the degradation state of a track component, characterized by some measures, is compared with a given set of thresholds on these measures which correspond to triggers of different maintenance actions, the timing and type of maintenance can be determined. The duration of a maintenance activity is generated from the relevant distribution function which is determined from historical records. At last, in conjunction with the cost for each action, obtained from market or records, the maintenance cost is obtained. 
The model is illustrated in Figure 2. Here it shows the modular structure of the model where the track of interest is separated into consecutive segments, from 1 to $\mathrm{n}$. Maintenance cost is then calculated on a segment-basis before they are aggregated into the overall maintenance cost for the whole track. Also shown in Figure 2 is the application scope of opportunistic maintenance. Here opportunistic maintenance refers to maintenance that takes the opportunity of working on one type of track components from a segment to work on the same type of track components from other segments. For example, sleepers from different segments can be renewed together at the same time. Other maintenance types considered include: preventive, corrective and concurrent maintenance. Here concurrent maintenance differs from opportunistic maintenance on that different track components from a same segment are maintained at the same time. Preventive maintenance and corrective maintenance differ by the timing of application: before or when the system failure is incipient or has taken place. These three maintenance types are shown in Figure 3 as well as the composition of a segment model.

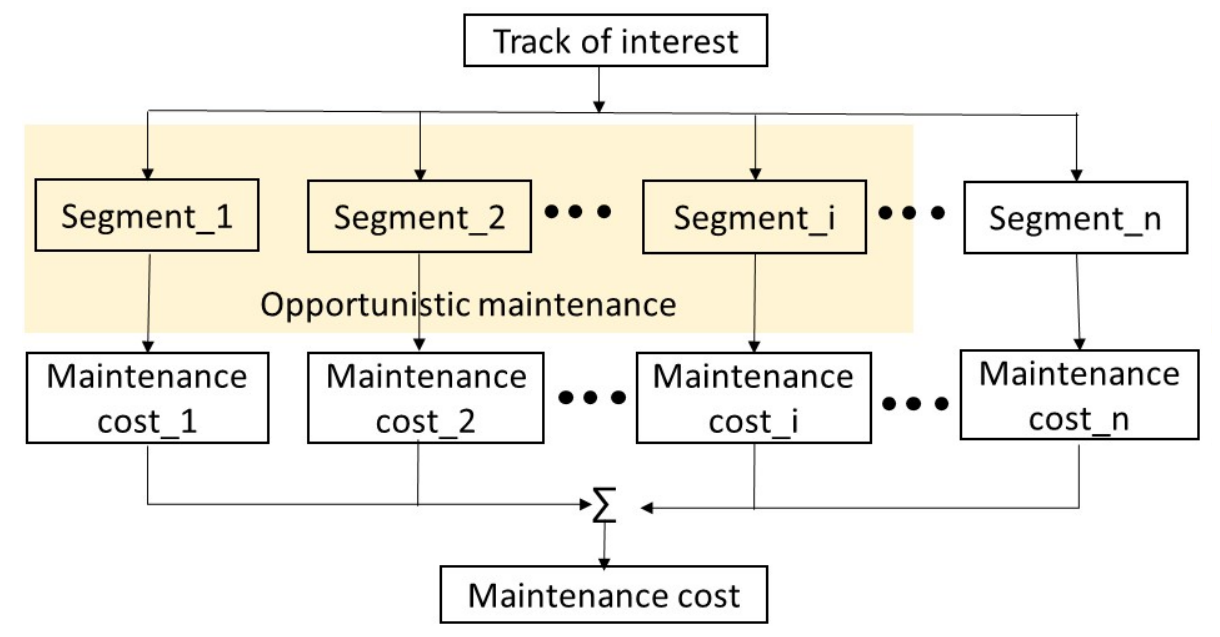

Figure 2. Structure of the proposed LCC model to estimate maintenance cost of a track

A typical ballated track with components: sleepers, ballast and rails, is used to illustrate the concept in Figure 3. The failures considered include: sleeper failures; rail Rolling Contact Fatigue and surface wear including corrugation, and track geometry deterioration. Track geometry condition is represented by longitudinal level and horizontal alignment for they are the most widely used indicators for initiating maintenance interventions. The maintenance actions considered include: sleeper replacement; ballast tamping and stone-blowing and ballast replacement; and, rail grinding. To apply the model to a slab track, the track composition needs to conform to the slab track configuration: sleeper as a component may not form part of the system and be removed. The concrete track slab, on the other hand, may be added. Degradation models for the added components, however, should be in place.

The structure shown in Figure 3 is similar to that by Michael (1985) but it is datadriven as opposed to the mechanistic approach Michael takes. As a result, uncertainties at each step of the model are allowed. In addition, during the modelling process, 
interactions between track components, between maintenance actions and track components, as well as various maintenance options, can be explored.

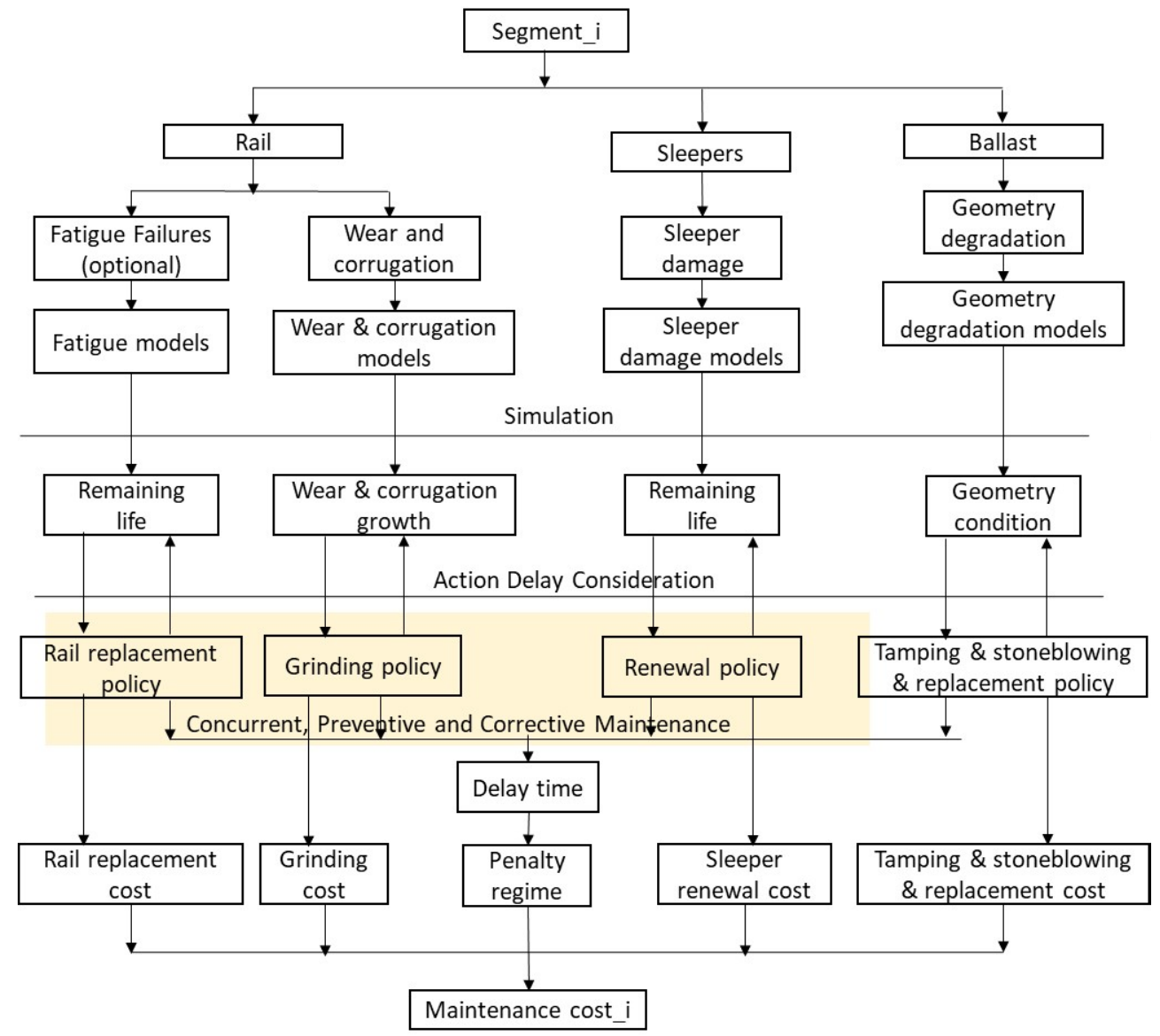

Figure 3. Structure of the proposed LCC model to estimate maintenance cost of a track segment

\section{Track geometry degradation model}

Separate degradation models are established for longitudinal level and horizontal alignment. Two maintenance actions, i.e. tamping and stone-blowing and their restorative effects are considered in each model. It is noted that stone-blowing cannot be followed by tamping. The fine particles injected during stone-blowing may slip into the voids of ballast particles, causing increased degradation rate of ballast and drainagerelated issues if tamping is still applied afterwards. It is also noted that track geometry degradation is only relevant to ballasted track as ballast is found to be the most significant contributor to track settlement (Selig and Waters, 1994).

Gamma process, by being non-increasing, is considered most suitable for modelling track geometry degradation between successive tamping interventions. The following assumptions are made with regard to the gamma process: 
1) $x_{i[t f t \cdot n]}=0$ with probability 1 (here time $t_{i n}$ is defined as the initiation time of degradation on segment $i . x$ is a measure of the degradation level, i.e. mean of the standard deviation of longitudinal level or horizontal alignment on a segment); and,

2) the increments of the measure over non-overlapping intervals are independent and identically distributed and follow the gamma distribution, i.e.

$$
D x_{i, j} \sim \mathrm{Ga}(D x \mid a, b)=\frac{b_{i}^{a_{i j}}}{G\left(a_{i j}\right)} D x^{a_{i j}-1} \mathrm{e}^{-b_{i} D x}
$$

where $a_{i i}$ and $b_{i}$ are the respective shape and rate parameter associated with the segment $i . j$ indexes the measurement time. $G$ is the gamma function. The mean and variance of $D x_{i, i}$ are given by $a_{i, i} / b_{i}$ and $a_{i, j}^{2} / b_{i}$, respectively.

The increments are then related to the actual size of the measure through

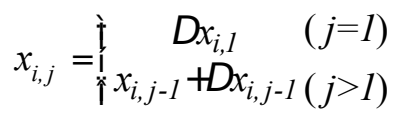

Based on the assumptions and the property of a gamma distribution, i.e. the sum of two gamma distributions sharing a same rate parameter generates a new gamma distribution of the same rate parameter and a shape parameter which equals the combination of the summands (Parzen, 1964, Section 1.1), i.e.

$$
x_{i, j}=\underset{k=j}{\stackrel{j}{a}} D x_{i, k} \sim \operatorname{Ga}\left(\stackrel{j}{\stackrel{j}{a}} a_{i k}, b_{i}\right)
$$

Assuming the shape parameter to have a form of

$$
a_{i j}=a\left(t_{j}-t_{i 0}\right)+b
$$

where $a$ and $b$ are related to the growth rate and the initial geometry degradation state of the corresponding track segment after a tamping or stone-blowing intervention, respectively. To correlate with the maintenance history, a power relationship is assumed between $a$ and the number of historical tamping or stone-blowing interventions, $N$, by

$$
a=q_{1}^{N}
$$

where $q_{1}$ is the parameter to be determined through simulation.

$b$ is assumed to correlate with $N$ through,

$$
b=q_{2}^{N}+e
$$

where the first term on the right hand side represents the initial condition at the finishing moment of a tamp. A random error componente is introduced to account for the variation of the initial condition across neighbouring segments. If the normal distribution is assumed for $\mathrm{e} \sim N\left(0, \mathrm{~S}^{2}\right), \mathrm{S}$ can be determined through simulation based on historical data.

It is noted that other growth laws between $a_{i i}$ and time can also be assumed, such as exponential and logarithmic. Different relations can also be assumed between $a, b$ and the number of the historical number of interventions $N$. The exponential relation is assumed here to account for the fact the effectiveness of the maintenance action diminishes with time gradually, to which an exponential relation seems proper. The best representative relation has to be determined by the data.

The data, or the measured longitudinal level and horizontal alignment data, $\boldsymbol{y}$, are related to the actual condition of the geometry measures, $\boldsymbol{x}$, by:

$$
y=p x+q+e
$$


where $\boldsymbol{e}$ represents the random error from the measurement and may follow a multivariate normal distribution $\mathbf{e} \sim \boldsymbol{M V N}(\mathbf{0}, \mathbf{S})$ where $\boldsymbol{S}$ is the variance-covariance matrix; $\boldsymbol{p}$ and $\boldsymbol{q}$ represent the systematic error from measurements.

In order to account for both the individual segment behaviour and the cohort behaviour of neighbouring segments when degrading, the hierarchical Bayesian approach is used. By making $q_{1}, q_{2}$ and $s$ common to all the segments, i.e. the restoration effect of each maintenance action is consistent across segments, the interaction between segments is accounted for. By allowing $b_{i}$ to be unique to each segment, the difference across segments is preserved.

Using the training data, distributions of model parameters for the gamma process can be determined. Rather than developing sampling algorithms from scratch, tools such as OpenBUGS (Lunn et al., 2009) or Jags (Miyamoto, 2012) have been available. Once the model is known, in each simulation step, a sample for each model parameter can be generated, which can be used in Eqs. (3.6) and (3.7) to determine $a$ and $b$ and then in Eq. (3.5) to determine $a_{i, i}$. The averages of $D x_{i, i}$ and finally $x_{i, i}$ at each time step can be determined subsequently.

To determine the timing for tamping and stone-blowing, the thresholds for their actions are prescribed. In each time step of the simulation, these limit values are compared with the simulated track degradation level. Once exceeded, the associated action is actuated. Duration of a maintenance action is generated from the corresponding distribution, determined from the maintenance records. In combination with the labour cost rate for each maintenance as well as equipment and material cost, the segment maintenance cost is determined.

If ballast replacement can be determined by the track geometry condition, the timing for its action can be decided by comparing the simulated track geometry condition with the predefined threshold. Different with ballast tamping/stone-blowing maintenance ballast replacement is performed only once. After the operation, the track is restored to a condition as good as new. Other replacement rules can also be explored, such as the time interval between successive tamping or stone-blowing actions and the duration of a maintenance action.

\section{Sleeper degradation model}

The degradation model for sleepers determines the projected timing and frequency of sleeper renewals together with a given renewal policy. This part may not be relevant to slab tracks without sleepers or with sleepers but embedded in the concrete track slab.

The model is adapted from Yun and Ferreira (2003). It is based on the time to failure distributions of the sleepers. The following assumptions are made when establishing the model:

1) the time to failure for a sleeper follows the Weibull distribution,

2) sleeper failure is only considered to be stress dependent. In other words, other failure mechanisms such as spike killing (due to shrinkage of timber sleepers), plate cutting, biological decay for time sleepers (Zhang et al., 2000), and fastening system failures, are not considered, 
3) each sleeper degrades independently until fails when the stress level the neighbouring sleepers carry is increased.

The maintenance strategy investigated is that whenever there is a cluster of defective sleepers then renewal of the cluster is actuated immediately (or with some known time delay) (the number of cluster is to be specified according to the actual practice envisaged). But other maintenance policies, including the one based on the total number of sleeper failures on a section, can be equally examined by the model.

Following Yun and Ferreira (2003) three levels of stress are defined: 'under normal' where sleepers are all in good condition; 'normal' where one sleeper fails and 'high' where two or more adjacent sleepers fail at a similar time. As a result, three sets of parameters are defined for the underlying Weibull distribution. Two pertinent quantities are the life of a sleeper, $t$, and the remaining life of a sleeper after some time, $t_{0}$, has elapsed, $T$, which are given by,

$$
t=b(-\ln F)^{\frac{1}{a}}
$$

and,

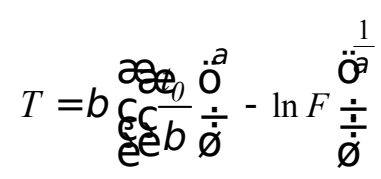

, respectively. Here $a$ and $b$ are the respective shape and scale parameters of the Weibull distribution and $F$ is a random value between 0 and 1 . In this model, each sleeper has a corresponding Weibull distribution. When the stress level a sleeper is subject to changes, the equivalent life and the remaining life should be re-calculated based on the corresponding Weibull distribution.

To implement the model,

1. record the number of sleepers, $N$, on a segment; specify the time step size for the simulation, $T_{\text {step }}$ (e.g., a year); use $T_{0 i}$ to record the age of each sleeper, $i=0,1,2, \mathrm{~L}, N$; assume $T_{0 i}=0$ at the beginning of the simulation run, i.e. each sleeper is new (actual ages may be used instead if available) and use $T_{\text {abs }}$ to record the elapsed time, $T_{\mathrm{abs}}=0$ at the start of the simulation run,

2. generate $N$ samples from the uniform distribution $(0,1)$, based on which and the determined Weibull distribution generate the life for each sleeper, according to Eq.(3.9),

3. locate sleepers whose remaining life, $T_{\text {remain }, i}$, is less than a year (or other defined values) or, whose life has already expired, $T_{0 i} £ T_{\text {abs }}$. If there is any failure, go to 4 ; otherwise, 6 ,

4. change the stress level of the neighbouring sleepers of the failed sleeper: one at the front and one at the back from 'under normal' to 'normal'; update the Weibull distribution with parameters appropriate for that stress level (if two or more consecutive failed sleepers are detected, change the stress level to 'high' and the parameters for the Weibull distribution accordingly for the sleepers before and after the failed cluster),

5. based on the modified model parameters, calculate the equivalent age, $t_{0 i}$, of each sleeper, $j$, in the neighbourhood, according to Eq.(3.9), i.e. equal to the life of the failed sleeper; and equate their recorded age $T_{0 i}$ to $t_{0 i}$, 
6. accumulate the simulation time by $T_{\text {abs }}=T_{\text {abs }}+T_{\text {step }}$, and the age of each sleeper by $T_{0 i}=T_{0 i}+T_{\text {step }}, i=0,1,2, \mathrm{~L}, N$,

7. determine the remaining life of each sleeper, $T_{\text {remain, },}$, by substituting $T_{0 i}$ as the elapsed time $t_{0}$ into Eq.(3.10), $i=0,1,2, \mathrm{~L}, N$,

8. repeat $3-7$,

9. whenever two or more consecutive sleepers fail together, renew them, and set the age of the renewed sleepers to zero, $T_{0 i}=0$ for the relevant $i$,

10. repeat 2-9 until end of the time horizon.

Different renewal policies can be conveniently investigated by changing the rule prescribed in step 8 .

The number of renewals per sleeper location and the total number of replacements are recorded during the simulation. The information is used to estimate the sleeper maintenance cost. In case the time spent on sleeper renewal is significant, the cost due to labour and service disruption needs to be updated. The effective sleeper usage and remaining life obtained in the simulation remain valid due to the likely stopped traffic and therefore usage of the sleepers over the period.

\section{Rail degradation models}

Three rail degradation mechanisms are considered: rolling contact fatigue, RCF; wear; and, rail corrugation. The pertinent maintenance actions are rail grinding and piece replacement. Slab track due to more uniform support stiffness is likely to be less affected by RCF than ballasted track.

Two types of failures may occur from RCF: failures of welds and failures of defects due to other causes including cracks. In both failure cases, the failed piece of rail needs to be replaced. A weld failure introduces an additional weld but a defect failure introduces two. The two failure types are considered jointly in the model.

The model is an adaptation of Zhao et al. (2006) with alterations to make it fit within the proposed LCC model framework in Figure 3. The following assumptions are made in the model:

1. the time to failure of all RCF-induced defects follows the Weibull distribution,

2. the emergence of defects on a section of track follows the non-homogeneous Poisson process, i.e. the arrival rate is time-dependent.

The parameters for the Weibull distribution and the intensity function of the Poisson process have to be inferred from historical records.

Differences with the model by Zhao et al. (2006) include:

1. both weld failures and defect failures are dealt with on a one-by-one basis, i.e. the collective number of failures at a time is generated by the simulation but not from direct statistical inference.

2. the effect of imperfect inspections on the overall number of defects is not considered. This is based on the fact that false detections, i.e. reporting non-existent defects as defects, and missing detections are un-avoidable during measurements. However, their occurrences are most likely only taking place when a defect has a size that is below or close to the resolution of the measurement device. The threat 
to the system integrity imposed is only secondary as a result. However, the observed number should be used to update the number generated by the simulation if available.

The steps to implement the model are:

1. record the number of welds, $M$, and the number of defects, $N$, on the segment of interest; specify the time step size for the simulation, $T_{\text {step }}$ (e.g., a year); assume the initial age of each weld and defect as $T_{0 i}=0$ and use $T_{\text {abs }}$ to record the elapsed simulation time, $T_{\mathrm{abs}}=0$ at the start of the simulation run,

2. sample $(M+N)$ values from the uniform distribution $(0,1)$ and use them to generate the life of each of the $M$ welds and the $N$ defects, according to Eq.(3.9),

3. locate the weld/defect whose remaining life, $T_{\text {remain, },}$, is less than a year (or any other defined values), or, whose life has expired, $T_{0 i} £ T_{\text {abs }}$. If there is any failure, go to 4; otherwise, 7 ,

4. if it is a weld failure, renew the weld by changing its age, $T_{0 i}$, to 0 and add one new weld to the population of welds, with age 0 ; if it is a defect failure, add two new welds to the population of defects, each with an age of 0 ,

5. generate a number of defects based on the determined arrival rate of the assumed non-homogeneous Poisson process for the defect generation process and assume all their ages equal to $T_{\text {step }}$; and, add them to the population of defects,

6. update the age of each weld and each defect, $T_{0 i}=T_{0 i}+T_{\text {step }}$; and, accumulate the elapsed simulation time $T_{\text {abs }}=T_{\text {abs }}+T_{\text {step }}$,

7. determine the remaining life of each weld and each defect, $T_{\text {remain, },}$, by substituting $T_{0 i}$ as the elapsed time $t_{0}$ into the corresponding Eq.(3.10) determined separately for welds and defects,

8. repeat 3 to 7 till end of the time horizon.

If an inspection scheme is in place, at each scheduled inspection time, the number of the welds and defects may be reduced by a factor to account for the increased knowledge. This factor, however, has to be learnt from actual observations. A series of values can be tested to investigate the impact of inspections by the model. A real world inspection requires a restart of the simulation.

The number of weld and defect failures is tallied during the simulation, based on which the maintenance cost is estimated. If grinding is considered, the life time of each weld and each defect is assumed to be improved after each grinding and this effect is accounted for by a correction factor, as used by Zhao et al. (2006).

The modelling for rail wear and corrugation growth is similar to that for track geometry degradation. Gamma process is considered most appropriate to represent the process for its monotonically increasing nature in the absence of external interferences, e.g., rail surface grinding. The procedure developed for track geometry degradation modelling is equally applicable. The wear and corrugation data, usually in terms of sizes of the irregularities, are desirable inputs to the model. However, parameters resulting from wear and corrugation can also be representative, such as the associated noise and vibration, for they are often linearly correlated with the level of rail surface irregularities. A set of rules, analogous to those for the track geometry monitoring, are required to initiate maintenance actions, including grinding. The number of maintenance actions and possibly the duration of each action when significant, as outputs from the model, give rise to the associated maintenance cost in combination 
with known cost rates. In case no data is inaccessible but a prescribed grinding scheme, LCC is accumulated based on the maintenance plan. Mitigation measures including grinding can have a significant impact on rail surface wear and corrugation. To account for the effect during the simulation, a factor, between 0 and 1 , may be applied to the simulated irregularity sizes. The factor has to be determined based on field measurement records. In real applications to guide maintenance planning, each update on the sizes of wear and corrugation areas requires a restart of the simulation.

\section{User cost model}

User cost may result from either speed restriction or line closure, due to extensive maintenance work on the affected track. To account for this cost element, a set of rules for triggering speed restriction or line closure should be developed, similar to those designed for the various maintenance actions but with more stringent conditions. The distribution of time spent on each type of service disruption interventions is determined from historical records. Alternatively, the work rate and the mobilization/demobilization time can be estimated and used to estimate the necessary track possession time reversely. During the simulation run, whenever conditions for a service disruption are met, a duration is generated from the corresponding distribution function. The simulation time is incremented by the same amount then. Accumulating all of the delay time, together with the cost rate, leads to the user cost.

\section{Coordination of maintenance actions}

Coordination of maintenance on various track components is enabled during the simulation run time. This is achieved through comparing the degradation level of each component against criteria set out for triggering the various maintenance actions. Four interrelated maintenance strategies are considered in the model: preventive and corrective, opportunistic and concurrent. A clear set of rules for each maintenance solution is required to initiate their implementations. An exemplar set of rules is listed below which is subject to further improvement:

1) Preventive maintenance

For all of the track components and maintenance rules, preventive maintenance is triggered by setting a threshold which is lower than the otherwise normal maintenance threshold, i.e. earlier occurance than corrective maintenance.

For example:

- Sleepers: this is defined as the length of the remaining life of a sleeper, say, less than 1.5 years instead of one year which is set for the renewal.

- Longitudinal level and horizontal alignment: the Standard Deviations are set blow those for the normal track geometry correction action, e.g., $80 \%$ of the normal thresholds.

- Ballast tamping/stone-blowing: the Standard Deviations of the longitudinal level or the horizontal alignment are set at $75 \%$ of the thresholds under normal conditions.

- Rail welds: the remaining life of a weld is less than 3 years.

2) Corrective maintenance 
This corresponds to the normal maintenance decision: action is only taken when the failure is detected or the thresholds to initiate an action have been reached. Such rules include:

- Two or more consecutive sleepers have failed.

- Track geometry has deteriorated to a level above a given threshold.

3) Opportunistic maintenance and Concurrent maintenance

The criteria set for both the preventive maintenance and corrective maintenance are also applicable under these two strategies but same components of different segments or different components of a same segment may be of concern.

4) Speed restriction and line closure

Such rules may include:

- over a number of consecutive sleepers have failed at a time

- the track geometry deteriorates to an unsafe state to continue normal operation

- over a number of welds have failed.

LCC Model implementation

To unify the separate component-based models and the various maintenance strategies under the proposed framework, the procedure below needs to be followed when implementing the model:

1. Segment the track under investigation based on predefined rules and collect data from each segment accordingly;

2. Determine the degradation models for the major track components in each segment:

- For the track geometry degradation modelling, separate sets of model parameters for the gamma processes used to represent the longitudinal level and horizontal alignment growth processes are determined based on historical records.

- For sleeper degradation modelling, the shape and scale parameters of the assumed Weibull distribution are determined based on historical records.

- For rail weld degradation modelling, the scale and shape parameters of the assumed Weibull distribution are determined from the historical records.

- For other rail surface defect degradation modelling, the generation rate of new defects and the time-to-failure distribution are determined based on historical records or expert judgement.

3. Draw a sample from each of the distributions determined in 2 to start the simulation;

4. Simulate the degradation state of each track component per specified time increment step:

a. Compare the degradation levels with the corresponding predefined maintenance thresholds to identity the necessity and opportunity of taking maintenance actions at the time step.

b. Record the number of the maintenance actions and types for each track component.

c. Generate a delay time according to the type of a maintenance action and accumulate them separately.

d. Compare maintenance cost estimated from the maintenance actions with the available budget:

i. If the budget is abundant, the planned maintenance interventions proceed; 
ii. Otherwise, prioritize the maintenance tasks and proceed only with the high priority ones. Any backlog resulted from a component will be accounted for by an increased degradation rate or reduced life in the subsequent simulation steps for that component;

5. Iterate the steps 3-4 until the end of the specified time horizon;

6. Calculate LCC based on the total numbers of various maintenance actions and also the delay times (user cost); and,

7. Repeat 1-6 sufficient times till statistically important results are generated for LCC and other parameters.

\section{Discussion}

The outstanding questions left unanswered by the model are addressed here. These include the unaccounted track components, such as fastening systems, switches and concrete track slabs; the unaccounted maintenance actions including inspections and derailments as well as interaction between different track components.

In this work, a simulation-based approach has been adopted to describe degradation of track components in the model proposed. Through this approach, all of the track components can be incorporated under the same framework. Different maintenance strategies can be explored. However, one caveat of applying data-driven only approaches is that connection with the physical world is lost, i.e. reliability of the results is totally dependent on the quality of the input data. A hybrid approach which can leverage the merits of both statistical and mechanistic models may help but this area is rarely explored.

Some track components have not so far been included in the model, in particular, the fastening systems; switches; support structures and concrete track slabs for slab track constructions. For fastening system and switches, no proper degradation model is found in the literature, which may be due to lack of proper indicators to represent their degradation states. Statistical approaches may be used in these cases by determining the time-to-failure distribution for each type of the systems. To account for the maintenance and renewal cost of a system only, the number of maintenance and replacement interventions and the cost of each action are sufficient, though the cost is dependent on usage and may not be representative across different sites. Intensive research has been conducted on understanding the failure mechanisms of concrete track slabs, particularly crack detection, e.g., Zhu et al. (2019) and measurement e.g., Saeed et al. (2019), but the control measures and action initiation criteria are not as fully developed as those for ballasted track. Gamma process is considered able to represent the crack development process. Together with the set maintenance thresholds it allows the exploration of the performance of various counter measures.

An appropriate measure that can characterise usage of a track component also needs to be chosen when determining the time-to-failure distribution for that component. Calendar time may not be sufficient, especially when there is significant change of traffic/operation conditions during the lifetime of that component such as increased 
load and speed. Other measures such as tonnes, tonnes x number of wheel passes, operation time, and tonne-km may be more representative.

Unplanned maintenance and the associated cost need to be considered separately from the model. According to Zoeteman (2001), minor maintenance activities can be evaluated by using Failure Mode Evaluation Analysis (FMEA) tool. The associated cost may be determined based on the volume of such maintenance. Major accidents, including derailment, though rarely take place, can be costly and time-consuming to fix. The random nature of such events can only be learnt from historical data where the occurance frequency of derailments can be derived. The associated cost can then be established.

Inspections need to be differentiated according to its type, i.e. time-dependent or condition-based. For time-dependent inspections, the cost can be determined from the frequency of applications and the cost per inspection. For condition-based inspections, the threshold for instigating such an inspection needs to be known. Similar to determine the timing of a maintenance action, a known threshold is used to decide the timing of an inspection during the simulation run by comparing it with the predicted component degradation state. To assess the impact of an inspection on revealing the degradation state of the affected track components, a correction factor may be devised to represent the increased knowledge.

The effect due to the interaction between different track components can be difficult to quantify in practice, for example, the impact of a sleeper damage on subsequent track geometry degradation. A correction factor may be introduced. A sensitivity analysis may also be conducted by using a series of improvised values for the correction factor in the developed model to test changes of the timings of following track maintenance actions. Field tests may be arranged to validate such studies.

Finally, to implement the simulation-based LCC model, a significant amount of data is required. The inputs include track geometry data, maintenance history, sleeper life time data, rail RCF and weld-failure history and others. Compiling the data from various sources may be necessary. To establish the robustness of the model further requires application of the model to a series of scenarios and validate the informed decisions with practice. This work is still ongoing.

\section{Conclusion}

A segment-based modular LCC model is proposed which allows various maintenance strategies to be modelled. It is component-based and allows components to be introduced or removed in order to fit a specific track configuration. Maintenance only takes place when a certain condition is met. Different maintenance strategies on different track components across different segments can be explored. As a result, the generated LCC is an unbiased evaluation of the performance of a track system.

To implement the various track degradation models, either fundamental Monte Carlo simulation or available packages is required in order to determine the model parameters and in the subsequent maintenance decision determination simulation. As a result, compared to Petri Net-based models, the proposed model is easier to implement while still allowing a detailed view of track degradation state at individual component level.

Three cost elements are considered in the model: construction cost, maintenance cost and user cost. Other elements are considered secondary but can be conveniently 
included when required. Budget constraints are enabled during the simulation run time which are represented by backlogs on components with low maintenance priorities.

\section{Acknowledgement}

This work is financially supported by the Australasian Centre for Rail Innovation under project PF15.

\section{References}

Ahac, M. \& Lakušić, S. 2015. Tram track maintenance-planning by gauge degradation modelling. Transport, 30, 430-436.

Al-Amin, M., Zhou, W., Zhang, S., Kariyawasam, S. \& Wang, H. 2014. Hierarchical Bayesian Corrosion Growth Model Based on In-Line Inspection Data. Journal of Pressure Vessel Technology, 136, 041401-041401.

Andrade, A. R. \& Teixeira, P. F. 2011. Uncertainty in Rail-Track Geometry Degradation: Lisbon-Oporto Line Case Study. Journal of Transportation Engineering, 137, 193-200.

Andrade, A. R. \& Teixeira, P. F. 2013. Hierarchical Bayesian modelling of rail track geometry degradation. Proceedings of the Institution of Mechanical Engineers, Part F: Journal of Rail and Rapid Transit, 227, 364-375.

Andrade, A. R. \& Teixeira, P. F. 2015. Statistical modelling of railway track geometry degradation using Hierarchical Bayesian models. Reliability Engineering \& System Safety, 142, 169-183.

Andrews, J. 2013. A modelling approach to railway track asset management. Proceedings of the Institution of Mechanical Engineers, Part F: Journal of Rail and Rapid Transit, 227, 56-73.

Caetano, L. F. \& Teixeira, P. F. 2015. Optimisation model to schedule railway track renewal operations: a life-cycle cost approach. Structure and Infrastructure Engineering, 11, 1524-1536.

Dahlberg, T. 2001. Some railroad settlement models-A critical review. Proceedings of the Institution of Mechanical Engineers, Part F: Journal of Rail and Rapid Transit, 215, 289-300.

Esveld, C. Computer-aided maintenance and renewal of track. ASME/IEEE Joint Conference on Railroads, 17-19 April 1990 1990. 165-170.

Fabrycky, W. J. \& Blanchard, B. S. 1991. Life-cycle cost and economic analysis, Englewood Cliffs, New Jersey, Prentice Hall.

Garry Thuer \& Vocks, H. 2012. Track slab system technology in Australia-innovation challenge based on European long term experiences. AusRail 2012. Canberra, Australia.

Guler, H. 2014. Prediction of railway track geometry deterioration using artificial neural networks: a case study for Turkish state railways. Structure and Infrastructure Engineering, 10, 614-626.

Guler, H., Jovanovic, S. \& Evren, G. 2011. Modelling railway track geometry deterioration. Proceedings of the Institution of Civil Engineers - Transport, 164, 6575.

Haigermoser, A., Eickhoff, B., Thomas, D., Coudert, F., Grabner, G., Zacher, M., Kraft, S. \& Bezin, Y. 2014. Describing and assessing track geometry quality. Vehicle System Dynamics, 52, 189-206.

Iso 15686-5 2017. Buildings and constructed assets-Service life planning-Part 5: Life cycle costing. 
Kilsby, P., Remenyte-Prescott, R. \& Andrews, J. 2019. A Petri Net-based life cycle cost analysis approach. Proceedings of the Institution of Mechanical Engineers, Part F: Journal of Rail and Rapid Transit, 233, 90-102.

Lasisi, A. \& Attoh-Okine, N. 2018. Principal components analysis and track quality index: A machine learning approach. Transportation Research Part C: Emerging Technologies, 91, 230-248.

Lunn, D., Spiegelhalter, D., Thomas, A. \& Best, N. 2009. The BUGS project: Evolution, critique and future directions. Statistics in Medicine, 28, 3049-3067.

Maes, M. A. 2013. Corrosion Growth Models and ILI-Based Estimation Procedures for Reliability-Based and Deterministic Pipeline Integrity Assessments. Phase 1: Formulation of Probabilistic Corrosion Growth Models and Corrosion Growth Rate Estimation. Contract PR-328-133600. Blade Energy Partners.

Meier-Hirmer, C., Riboulet, G., Sourget, F. \& Roussignol, M. 2009. Maintenance optimization for a system with a gamma deterioration process and intervention delay: Application to track maintenance. Proceedings of the Institution of Mechanical Engineers, Part O: Journal of Risk and Reliability, 223, 189-198.

Meier-Hirmer, C., Senee, A., Riboulet, G., Sourget, F. \& Roussignol, M. 2006. A decision support system for track maintenance. Compters in railways, X, 217-226.

Michael, B. H. 1985. Track maintenance cost analysis: An engineering economics approach, Washington, D.C., USA, Transportation Research Record 1029, Transportation Research Board, National Research Council, National Academy Press.

Milford, R. L. \& Allwood, J. M. 2010. Assessing the CO2 impact of current and future rail track in the UK. Transportation Research Part D: Transport and Environment, 15, 61-72.

Miyamoto, J. 2012. Functions for Working with OpenBUGS, WinBUGS \& JAGS Output.

Parzen, E. 1964. Stochastic Processes, San Francisco, Holden-Day.

Pratico, F. G. \& Giunta, M. 2018. LCC-based appraisal of ballasted track and slab tracks: limits and potential. The Baltic Journal of Road and Bridge Engineering, 13.

Prescott, D. \& Andrews, J. 2013. A track ballast maintenance and inspection model for a rail network. Proceedings of the Institution of Mechanical Engineers, Part O: Journal of Risk and Reliability, 227, 251-266.

Prescott, D. \& Andrews, J. 2015. Investigating railway track asset management using a Markov analysis. Proceedings of the Institution of Mechanical Engineers, Part F: Journal of Rail and Rapid Transit, 229, 402-416.

Quiroga, L. M. \& Schnieder, E. 2012. Monte Carlo simulation of railway track geometry deterioration and restoration. Proceedings of the Institution of Mechanical Engineers, Part O: Journal of Risk and Reliability, 226, 274-282.

Rama, D. \& Andrews, J. D. 2016. Railway infrastructure asset management: the whole-system life cost analysis. IET Intelligent Transport Systems, 10, 58-64.

Saeed, N., Abdulrahman, Y., Amer, S. \& Omar, M. A. 2019. Experimentally validated defect depth estimation using artificial neural network in pulsed thermography. Infrared Physics \& Technology, 98, 192-200.

Selig, E. T. \& Waters, J. M. 1994. Track geotechnology and substructure management, Lond, Thomas Telford Publications.

Shafahi, Y. \& Hakhamaneshi, R. 2009. Application of a Maintenance Management Model for Iranian Railways Based on the Markov Chain and Probabilistic Dynamic Programming. Transaction A: Civil Engineering, 16, 87-97. 
Sharma, S., Cui, Y., He, Q., Mohammadi, R. \& Li, Z. 2018. Data-driven optimization of railway maintenance for track geometry. Transportation Research Part C: Emerging Technologies, 90, 34-58.

Swei, O., Gregory, J. \& Kirchain, R. 2017. Probabilistic Approach for Long-Run Price Projections: Case Study of Concrete and Asphalt. Journal of Construction Engineering and Management, 143, 05016018.

Vale, C., Ribeiro, I. M. \& Calçada, R. 2012. Integer Programming to Optimize Tamping in Railway Tracks as Preventive Maintenance. Journal of Transportation Engineering, 138, 123-131.

Van Noortwijk, J. M. 2009. A survey of the application of gamma processes in maintenance. Reliability Engineering \& System Safety, 94, 2-21.

Van Noortwijk, J. M., Dekker, R., Cooke, R. M. \& Mazzuchi, T. A. 1992. Expert judgment in maintenance optimization. IEEE Transactions on Reliability, 41, 427432.

Yianni, P. C., Neves, L. C., Rama, D. \& Andrews, J. D. 2018. Accelerating Petri-Net simulations using NVIDIA Graphics Processing Units. European Journal of Operational Research, 265, 361-371.

Yun, W. Y. \& Ferreira, L. 2003. Prediction of the demand of the railway sleepers: A simulation model for replacement strategies. International Journal of Production Economics, 81-82, 589-595.

Zhang, S. 2014. Development of Probabilistic Corrosion Growth Models with Applications in Integrity Management of Pipelines. Doctor of Philosophy, University of Western Ontario.

Zhang, S. \& Zhou, W. 2015. Probabilistic characterisation of metal-loss corrosion growth on underground pipelines based on geometric Brownian motion process. Structure and Infrastructure Engineering, 11, 238-252.

Zhang, S., Zhou, W., Al-Amin, M., Kariyawasam, S. \& Wang, H. 2014. TimeDependent Corrosion Growth Modeling Using Multiple In-Line Inspection Data. Journal of Pressure Vessel Technology, 136, 7 pages.

Zhang, S., Zhou, W. \& Qin, H. 2013. Inverse Gaussian process-based corrosion growth model for energy pipelines considering the sizing error in inspection data. Corrosion Science, 73, 309-320.

Zhang, Y.-J., Murray, M. \& Ferreira, L. 2000. Modelling rail track performance: an integrated approach. Proceedings of the Institution of Civil Engineers - Transport, 141, 187-194.

Zhao, J., Chan, A. H. C., Roberts, C. \& Stirling, A. B. 2006. Assessing the Economic Life of Rail Using a Stochastic Analysis of Failures. Proceedings of the Institution of Mechanical Engineers, Part F: Journal of Rail and Rapid Transit, 220, 103-111.

Zhu, W.-F., Chen, X.-J., Li, Z.-W., Meng, X.-Z., Fan, G.-P., Shao, W. \& Zhang, H.-Y. 2019. A SAFT Method for the Detection of Void Defect inside a Ballastless Track Structure Using Ultrasonic Array Sensors. Sensors, 19.

Zoeteman, A. 2001. Life cycle cost analysis for managing rail infrastructure-concept of a decision support system for railway design and maintenance. European Journal of Transport and Infrastructure Research, 1, 391-413. 